\title{
Papel do enfermeiro na inserção dos parceiros no pré-natal e tratamento de gestantes com sífilis
}

\author{
Role of nurses in the insertion of partners in prenatal and treatment of pregnant women \\ with syphilis \\ El papel de las enfermeiras em lainserción de parejas em la atención prenatal y el \\ tratamiento de mujeres embarazadas con sífilis
}

Camila Rocha Silveira $^{1 *}$, Letícia Falkenbach da Costa $^{2}$, Morgana Thais Carollo Fernandes ${ }^{3}$, Raquel Malta Fontenele ${ }^{1}$.

\begin{abstract}
RESUMO
Objetivo: Identificar o papel do enfermeiro na inserção do parceiro no pré-natal e no tratamento de gestantes com exames positivos para sífilis. Métodos: Trata-se de uma revisão de literatura dos últimos dez anos, nas bases de dados: MEDLINE, LILACS, BDENF inseridos na BVSALUD; utilizando os descritores Sífilis; gravidez; recémnascido; parceiros sexuais; enfermagem; e terapêutica, cruzados por meio do conector booleano and e or. Resultados: Foram incluídos 22 artigos disponíveis na integra e nos últimos dez anos de pesquisa e publicação, nos idiomas português, inglês e espanhol. Considerações finais: $O$ enfermeiro tem um papel fundamental no acompanhamento e tratamento das gestantes com sífilis, além de que, o pré-natal das gestantes com exames de sífilis positivos é mais efetivo se houver a inclusão do parceiro. É necessário que seja realizado mais estudos sobre o papel do enfermeiro na abordagem dos parceiros das gestantes com sífilis.
\end{abstract}

Palavras-chave: Sífilis, Sífilis congênita, Gravidez, Parceiros sexuais, Enfermagem.

\section{ABSTRACT}

Objective: To identify the role of the nurse in the insertion of the partner in prenatal care and in the treatment of pregnant women with positive tests for syphilis. Methods: It is a literature review of the last ten years, in the databases: MEDLINE, LILACS, BDENF inserted in BVSALUD; using the descriptors syphilis; pregnancy; newborn; sexual partners; nursing; and therapeutics, crossed by means of the boolean connector and and OR. Results: 22 articles were fully available and in the last ten years of research and publication, in Portuguese, English and Spanish. Final considerations: The nurse has a fundamental role in the monitoring and treatment of pregnant women with syphilis, in addition to that, the prenatal care of pregnant women with positive syphilis tests is more effective if the partner is included. It is necessary to carry out further studies on the role of nurses in approaching the partners of pregnant women with syphilis.

Keywords: Syphilis, Congenital syphilis, Pregnancy, Sexual partners, Nursing.

\section{RESUMEN}

Objetivo: Identificar el papel de la enfermera en la inserción de la pareja en la atención prenatal y en el tratamiento de mujeres embarazadas con pruebas positivas de sífilis. Métodos: Esta es una revisión de la literatura de los últimos diez años, en las bases de datos: MEDLINE, LILACS, BDENF insertada en BVSALUD; utilizando los descriptores sífilis; el embarazo; recién nacido; parejas sexuales; enfermería; y terapéutica, cruzada por medio del conector booleano and y OR. Resultados: 22 artículos estuvieron completamente disponibles y en los últimos diez años de investigación y publicación, en portugués, inglés y español. Consideraciones finales: La enfermera tiene un papel fundamental en el monitoreo y el tratamiento de las mujeres embarazadas con sífilis, además de eso, la atención prenatal de las mujeres embarazadas con pruebas de sífilis positivas es más efectiva si se incluye a la pareja. Es necesario realizar más estudios sobre el papel de las enfermeras para acercarse a las parejas de mujeres embarazadas con sífilis.

Palabras clave: Sífilis, Sífilis congénita, Embarazo, Parejas sexuales, Enfermería.

${ }^{1}$ Centro Universitário Ritter dos Reis (UniRitter), Porto Alegre - RS.

*E-mail: camilarocha_silveira@hotmail.com

2 Universidade Luterana do Brasil (ULBRA), Canoas - RS.

${ }^{3}$ Universidade Federal do Rio Grande do Sul (UFRGS), Porto Alegre - RS.

SUBMETIDO EM: 8/2020 | ACEITO EM: 9/2020 | PUBLICADO EM: 11/2020 


\section{INTRODUÇÃO}

A sífilis é um agravo infectocontagioso, e suas principais formas de transmissão são por via sexual (anal, oral ou vaginal) e por via transplacentária em que a mãe infectada, que não realizou o tratamento, ou que o fez de forma incorreta, transmite para o feto o vírus treponema pallidum, sendo considerado um caso sífilis congênita (AVELLEIRA JCR e BOTTINO G., 2006; SECRETARIA DE ESTADO DA SAUDE SES-SP, 2008).

Segundo CAMPOS ALA, et al. (2012); a transmissão da sífilis congênita, conhecida como transmissão vertical, pode acontecer no parto ou em qualquer momento da gestação, dependerá do quão precocemente é feito o tratamento e do estágio da doença materna. A sífilis gestacional tratada de forma incompleta pode causar abortamento, morte fetal e morte neonatal (MACEDO VC, et al,2017; NASCIMENTO MI, et al, 2012).

As gestantes que não fizeram o tratamento ou fizeram de forma inadequada podem ter seus filhos assintomáticos, o que pode resultar em a não realização do diagnóstico, e, consequentemente, ao não tratamento, sendo não realizado o que é preconizado pelo Ministério da Saúde (MS). Conforme as Diretrizes para o Controle da Sífilis Congênita, do MS (2006); a criança assintomática com a mãe inadequadamente tratada, deve mesmo assim receber a terapêutica recomendada e acompanhamento após o tratamento.

Para fins de investigação clínica, as características principais da sífilis congênita são a prematuridade, lesões cutâneas, baixo peso ao nascer, icterícia, sofrimento respiratório, alteração liquórica e alteração nos exames radiológicos (MACEDO VC, et al, 2017; BRASIL, 2006).

De acordo com o Boletim Epidemiológico da Sífilis, divulgado pelo MS, no Brasil (2018), foram notificados 24.303 casos de sífilis congênita em neonatos e 206 óbitos por sífilis congênita em menores de um ano registrados em 2017 no Brasil.

Sendo $93,2 \%$ classificados como sífilis congênita recente, $3,5 \%$ casos de aborto por sífilis e 3,1\% casos de natimortos. Entre os anos de 1998 a 2004, o número de óbitos declarados por sífilis congênita foram 671 casos registrados no Brasil, com aumento significativo ao longo do tempo, com registro de 1.647 casos entre 2004 a 2017 (BRASIL, 2018).

A sífilis congênita é um problema de saúde pública que possui métodos de diagnósticos simples, tratamento barato e que pode ser evitada (CAMPOS ALA, et al., 2012; MACEDO VC, et al.,2017; VASCONCELOS MIO, et al., 2016).

O primeiro passo para a prevenção é a inclusão da gestante aos serviços de saúde para que ela possa realizar o pré-natal (VASCONCELOS MIO, et al., 2016). Destaca-se que durantes as consultas de pré-natal devem ser solicitadas os exames sorológicos, realizar a abordagem e orientação quanto ao tratamento e a inserção do parceiro sexual (CARDOSO ARP, et al., 2018; FIGUEIREDO MSNG, et al., 2015).

No entanto, ainda há um grande número de gestantes infectadas que não são orientadas corretamente em relação ao controle e prevenção da transmissão vertical da sífilis (MAGALHÃES DMS, et al., 2013).Estudos apontam que os principais fatores relacionados ao aumento de casos de sífilis congênita, são: desconhecimento da gravidade da doença, pré-natal inadequado ou a não realização do mesmo, relaxamento das medidas preventivas por parte das equipes de saúde e promiscuidade sexual (CUNHA AE, et al., 2006).

Um pré-natal de qualidade deve ser aquele em que o parceiro é inserido e as orientações sobre os riscos relacionados à infecção pelo T. pallidum por meio da transmissão são esclarecidas, (principalmente para evitar reinfecção durante o tratamento) para que mulheres com sífilis e seu (s) parceiro (s) tenham práticas sexuais seguras durante o tratamento (FIGUEIREDO MSNG, et al., 2015; DOMINGUES RMSM, et al., 2014).

A participação do parceiro é de extrema importância, pois há a necessidade de ele fazer o tratamento completo junto com a gestante, para minimizar os riscos de transmissão vertical (FIGUEIREDO MSNG, et al., 2015). A escolha dessa revisão foi feita partir de uma experiência de estágio extracurricular, realizado em um hospital público de Porto Alegre, em que foram analisadas fichas de casos de sífilis em gestantes e sífilis congênita, e que, a partir disso, foi observado que na maioria dos casos os parceiros das gestantes não realizavam o tratamento, consequentemente tornando o tratamento da gestante inadequado. 
A sífilis gestacional é uma doença conhecida há anos e de fácil tratamento, porém, ainda há muita dificuldade no controle por parte dos profissionais de saúde, e com isso, as taxas de transmissão têm aumentado a cada ano, e a falta do tratamento do parceiro das gestantes pode ser um fator que aumente 0 número de casos da transmissão vertical. Portanto, o estudo tem como pretensão responder a seguinte pergunta de pesquisa: o que a literatura abrange sobre o papel do enfermeiro na inserção do parceiro no prénatal e no tratamento de gestantes com exames positivos para sífilis?

\section{MÉTODOS}

Trata-se de um artigo de revisão integrativa, que inclui a análise de pesquisas relevantes que dão suporte para a tomada de decisão e a melhoria da prática clínica, possibilitando a síntese do estado do conhecimento de um determinado assunto, além de apontar lacunas do conhecimento que precisam ser preenchidas com a realização de novos estudos (MENDES KDS, et al., 2008).

A busca bibliográfica foi realizada em março e abril de 2019, nas bases de dados: Medical LiteratureAnalysisandRetrieval System Online (MEDLINE), Literatura Latino-americana e do Caribe em Ciências da Saúde (LILACS), Base de dados em Enfermagem (BDENF) inseridos na Biblioteca Virtual de Saúde (BVSALUD).

Foi utilizada como estratégia de busca a combinação dos seguintes Descritores em Ciências da Saúde (DeCS): sífilis, gravidez, recém-nascido, parceiros sexuais, enfermagem, e terapêutica, cruzados por meio do conector booleano AND e OR.

Seguindo os critérios de busca, inicialmente foram identificados 104 artigos na base de dados. Foram excluídas 34 publicações após a leitura do título e resumo. Posteriormente, de acordo com o critério de exclusão e os duplicados, foram excluídos 48 artigos.

A amostra final foi composta de 22 artigos. Foram incluídos artigos disponíveis na íntegra, publicados em português, inglês e espanhol, com delimitação cronológica de pesquisa e de publicação realizadas nos últimos dez anos, que apresentassem informações sobre o papel do enfermeiro no tratamento da sífilis na gestação com inserção do parceiro.

Foram excluídos artigos que abordassem outras doenças sexualmente transmissíveis; teses, dissertações, relatos de experiência e estudos de caso. Os artigos incluídos nesta revisão foram lidos na íntegra e sumarizados por objetivos, ano de publicação, método, nível de evidência e principais resultados.

\section{RESULTADOS}

Apesar de haver bastante conteúdo sobre a sífilis, há poucos estudos que focam no papel do enfermeiro na inserção dos parceiros no pré-natal e no tratamento das gestantes com sífilis (Tabela 1). Dos 22 artigos selecionados, apenas 04 artigos tinham como foco principal esse assunto e 11 desses artigos incluídos abordavam os fatores sociodemográficos e comportamentais das gestantes com sífilis e seus parceiros. Alguns estudos abordavam assuntos distintos do foco principal tais como: 06 artigos sobre intervenção e tratamento e 1 artigo sobre sífilis falso positivo.

Tabela 1 - Relação das temáticas principais na pesquisa com a quantitativo de artigos.

\begin{tabular}{lc}
\hline Assunto principal & Quantitativo de artigos (n=22) \\
\hline $\begin{array}{l}\text { Fatores sociodemográficos e comportamentais das gestantes com } \\
\text { sífilis e seus parceiros; }\end{array}$ & $50 \%$ \\
\hline Intervenção e tratamento da sífilis gestacional; & $27,27 \%$ \\
\hline $\begin{array}{l}\text { O papel do enfermeiro na inserção dos parceiros no pré-natal e no } \\
\text { tratamento das gestantes com sífilis; }\end{array}$ & $18,18 \%$ \\
\hline Sífilis falso positivo (efeito prozona). & $4,54 \%$ \\
\hline
\end{tabular}

Fonte: Silveira CR, et al., 2020. 
Mesmo que poucos artigos tivessem como foco principal o papel do enfermeiro na inserção do parceiro no pré-natal e no tratamento da sífilis em gestantes, 10 estudos falaram da importância do tratamento do parceiro em casos de sífilis gestacional para o tratamento adequado da gestante e 11 dos artigos abordavam que a triagem da sífilis no pré-natal deve ser iniciada o quanto antes no primeiro trimestre de gestação com a abordagem do parceiro.

E, 07 artigos abordavam os eventos adversos na gestação causados pela sífilis. Em 04 dos artigos encontrados, foi relatado que os enfermeiros e profissionais de saúde possuem muita dificuldade em abordar os parceiros das gestantes com sífilis.

A relação dos principais resultados das pesquisas que auxiliam a responder o questionamento do presente estudo está na: (Tabela 2). Esses assuntos não se apresentam como assunto principal no artigo, mas sim em algum momento os autores pontuam a temática que atendeu ao critério de inclusão.

Tabela 2 - Relação dos assuntos abordados nos resultados das pesquisas com a quantidade de artigos.

Assunto abordado

Quantidade de artigos ( $n=22)$

Triagem da sífilis no pré-natal deve ser iniciada o quanto antes no primeiro trimestre de gestação com a abordagem do parceiro;

$50 \%$

Artigos que abordaram em algum momento a importância do tratamento do parceiro em casos de sífilis gestacional para o tratamento adequado da gestante;

$45,45 \%$

Eventos adversos na gestação causados pela sífilis;

$31,81 \%$

Estudos que abordaram em algum momento que os enfermeiros e profissionais de saúde possuem muita dificuldade em abordar os parceiros das gestantes com sífilis.

$18,18 \%$

Fonte: Silveira CR, et al., 2020.

As publicações são de estudos realizados nos seguintes locais: Brasil (10), nas regiões nordeste (6), sudeste (2), centro-oeste (1) e em todas as regiões (1). Foram encontrados estudos de outros países como a Argentina (1); Ghana (1); Estados Unidos (1); China (1); Alemanha (1); América Do Norte (1); e estudo com dados mundiais (2) e estudos sem local definido (4).

Quanto ao delineamento metodológico das publicações, foram identificados: revisão sistemática e metanálise (5), revisão sistemática de ensaios clínicos randomizados (1), estudos descritivos (2), estudos transversais (1), estudos transversais e descritivos (1), estudos quantitativos (1), estudos qualitativos (2), estudos caso controle (4), estudos de coorte retrospectivos (2) e estudos de coorte (3).

Em relação aos anos de publicação, nos anos de 2010, 2012 e 2019 houve publicação de um artigo em cada ano. E em 2011 houve dois artigos, cinco em 2013, quatro em 2014, cinco em 2015 e três em 2016. Identificou-se predominância de estudos com foco nos fatores sociodemográficos e comportamentais das gestantes com sífilis e seu parceiro.

Quanto ao nível de estudo e grau de recomendação, tem-se 05 estudos com nível I.A de evidência; 01 com nível III.C; 05 com nível IV.C; 04 com nível V.C e 07 com nível VI.D. A classificação e análise dos artigos inclusos na busca está na (Tabela 3). 
Quadro 3 - Consolidado de análise da busca conforme ano de publicação, autor, título, métodos, resultados, nível de evidência e grau de recomendação.

\begin{tabular}{|c|c|c|c|c|c|}
\hline № & $\begin{array}{c}\text { Autores/ } \\
\text { Ano de } \\
\text { publicaç } \\
\text { ão }\end{array}$ & Título da publicação & Método & Principais resultados/ Conclusões & $\begin{array}{c}\text { Nível de } \\
\text { evidência e grau } \\
\text { de } \\
\text { recomendação* }\end{array}$ \\
\hline 1 & $\begin{array}{l}\text { Oliveira } \\
\text { DR e } \\
\text { Figueired } \\
\text { o MSN. } \\
2011 .\end{array}$ & $\begin{array}{l}\text { Abordagem conceitual } \\
\text { sobre a sífilis na gestação } \\
\text { e o tratamento de } \\
\text { parceiros sexuais. }\end{array}$ & $\begin{array}{c}\text { Estudo } \\
\text { descritivo. }\end{array}$ & $\begin{array}{l}\text { O artigo abrange dados epidemiológicos acerca da sífilis congênita, e mostra o } \\
\text { desenvolver do controle da sífilis durante os anos. Além disso, mostra que o tratamento } \\
\text { da sífilis gestacional só é eficaz quando é realizado o tratamento do parceiro. Esse } \\
\text { estudo ressalta a importância da inserção do enfermeiro no rastreamento, controle da } \\
\text { sífilis gestacional e na educação em saúde dos parceiros sexuais das gestantes. }\end{array}$ & VI.D. \\
\hline 2 & $\begin{array}{l}\text { Macedo } \\
\text { VC, et al., } \\
2017 .\end{array}$ & $\begin{array}{l}\text { Fatores de risco para } \\
\text { sífilis em mulheres: } \\
\text { estudo caso-controle. }\end{array}$ & $\begin{array}{l}\text { Estudo de } \\
\text { caso } \\
\text { controle. }\end{array}$ & $\begin{array}{l}\text { Pesquisa que analisou fatores sociodemográficos e comportamentais das gestantes } \\
\text { com sífilis e seus parceiros. Mesmo que não tenham sido abordados os parceiros } \\
\text { sexuais das gestantes com sífilis nessa pesquisa, foi avaliado que a incidência de } \\
\text { infecções sexuais pode estar ligada a situações de vulnerabilidade. Ressalta também, } \\
\text { que a assistência pré-natal adequada pode reduzir os casos de sífilis durante a gravidez } \\
\text { com estímulos do uso de preservativos, e de tratamento adequado. }\end{array}$ & V.C. \\
\hline 3 & $\begin{array}{l}\text { Silva AM, } \\
\text { et } \\
\text { al.,2016. }\end{array}$ & $\begin{array}{l}\text { Factores asociados con } \\
\text { falla en el diagnóstico y } \\
\text { tratamiento de sífilis } \\
\text { materna. }\end{array}$ & $\begin{array}{l}\text { Estudo de } \\
\text { caso } \\
\text { controle. }\end{array}$ & $\begin{array}{l}\text { Analise do histórico das gestantes com e sem sífilis, avaliando os riscos de apresentar } \\
\text { algum tipo de falha no tratamento. Abrange o histórico das gestantes, como: ser } \\
\text { primigesta, histórico de aborto, outras infecções sexuais, entre outros. Medidas como } \\
\text { educação em saúde poderiam reduzir a gravidez na adolescência e o número de casos } \\
\text { de sífilis. }\end{array}$ & V.C. \\
\hline 4 & $\begin{array}{l}\text { Nonato } \\
\text { SM, et } \\
\text { al.,2015. }\end{array}$ & $\begin{array}{l}\text { Sífilis na gestação e } \\
\text { fatores associados à sífilis } \\
\text { congênita em Belo } \\
\text { Horizonte-MG, 2010- } \\
2013 .\end{array}$ & $\begin{array}{l}\text { Estudo de } \\
\text { coorte. }\end{array}$ & $\begin{array}{l}\text { Esse estudo mostra que a sífilis pode estar relacionada à baixa escolaridade da } \\
\text { gestante e do parceiro, vulnerabilidade social e início tardio do pré-natal. E que pode } \\
\text { haver falhas na assistência pré-natal no controle da sífilis, e indicam que há necessidade } \\
\text { de propor novos meios de reduzir a transmissão vertical da sífilis. }\end{array}$ & IV.C. \\
\hline 5 & $\begin{array}{l}\text { Dassah } \\
\text { ET, et al., } \\
2015 .\end{array}$ & $\begin{array}{l}\text { Factors associated with } \\
\text { failure to screen for } \\
\text { syphilis during antenatal } \\
\text { care in Ghana: a case } \\
\text { control study. }\end{array}$ & $\begin{array}{l}\text { Estudo } \\
\text { caso } \\
\text { controle. }\end{array}$ & $\begin{array}{l}\text { Estudo sobre fatores sociodemográficos de risco para as gestantes com sífilis, como: } \\
\text { pré-natal na rede privada (devido à ausência das carteirinhas de pré-natal), número de } \\
\text { consultas pré-natais, idade, escolaridade, entre outros. O estudo conclui que, seja } \\
\text { reforçado o rastreamento da sífilis da mãe, principalmente em instituições privadas, e } \\
\text { que o parceiro seja incluído na triagem da sífilis no pré-natal, para um controle eficaz na } \\
\text { prevenção da sífilis congênita. }\end{array}$ & V.C. \\
\hline 6 & $\begin{array}{l}\text { Domingue } \\
\text { s RMSM, } \\
\text { et al., } \\
2014 .\end{array}$ & $\begin{array}{l}\text { Prevalência de sífilis na } \\
\text { gestação e testagem pré- } \\
\text { natal: Estudo Nascer no } \\
\text { Brasil. }\end{array}$ & $\begin{array}{l}\text { Estudo de } \\
\text { coorte. }\end{array}$ & $\begin{array}{l}\text { A prevalência da sífilis é maior em gestantes com maior vulnerabilidade social. Além de } \\
\text { que, a triagem da sífilis é mais eficaz no serviço privado, mesmo elas não possuindo } \\
\text { carteirinha de pré-natal. Abrange a importância do tratamento do parceiro para evitar } \\
\text { reinfecção na gravidez e prevenção de novos casos. }\end{array}$ & IV.C. \\
\hline
\end{tabular}

REAS/EJCH | Vol.12(11) | e4741 | DOI: https://doi.org/10.25248/reas.e4741.2020 Página 5 de 12 


\begin{tabular}{|c|c|c|c|c|c|}
\hline № & \begin{tabular}{|c|} 
Autores/ \\
Ano de \\
publicaç \\
ão \\
\end{tabular} & Título da publicação & Método & Principais resultados/ Conclusões & $\begin{array}{c}\text { Nível de } \\
\text { evidência e grau } \\
\text { de } \\
\text { recomendação* }\end{array}$ \\
\hline 7 & $\begin{array}{l}\text { Martha } \\
\text { WFR, et } \\
\text { al., } 2015 .\end{array}$ & $\begin{array}{l}\text { Maternal titers after } \\
\text { adequate syphilotherapy } \\
\text { during pregnancy. }\end{array}$ & $\begin{array}{l}\text { Estudo } \\
\text { coorte } \\
\text { retrospect } \\
\text { ivo. }\end{array}$ & $\begin{array}{l}\text { Esse estudo aborda que o tratamento varia de acordo com o estágio da sífilis, e que a } \\
\text { taxa mais rápida de declínio foi na sífilis primária e secundária, porque o declínio } \\
\text { depende do estágio da doença e não do tratamento em si. Gestantes com sífilislatente } \\
\text { tiveram um declínio menor. }\end{array}$ & IV.C. \\
\hline 8 & $\begin{array}{l}\text { Liu LL, et } \\
\text { al., } 2014 .\end{array}$ & $\begin{array}{l}\text { Incidence and risk factors } \\
\text { for the prozone } \\
\text { phenomenon in serologic } \\
\text { testing for syphilis in a } \\
\text { large control. }\end{array}$ & $\begin{array}{l}\text { Estudo de } \\
\text { coorte } \\
\text { retrospect } \\
\quad \text { ivo. }\end{array}$ & $\begin{array}{l}\text { Esse estudo fala do fenômeno da prozona, mais conhecido como falso positivo da sífilis. } \\
\text { Eles acreditam que esse fenômeno ocorre por um maior número de anticorpos. A sífilis } \\
\text { falso positivo pode ter resultados enganosos, e que dificultam o controle da sífilis } \\
\text { congênita. }\end{array}$ & IV.C. \\
\hline 9 & $\begin{array}{l}\text { Figueired } \\
\text { o MSNG, } \\
\text { et al., } \\
2015 .\end{array}$ & $\begin{array}{l}\text { Percepção de } \\
\text { enfermeiros sobre a } \\
\text { adesão ao tratamento dos } \\
\text { parceiros de gestantes } \\
\text { com sífilis. }\end{array}$ & $\begin{array}{l}\text { Estudo } \\
\text { Qualitativ } \\
\quad 0 .\end{array}$ & $\begin{array}{l}\text { Esse estudo mostra o quão importante é a qualificação do enfermeiro para a abordagem } \\
\text { dos fatores que interferem no tratamento do parceiro sexual das gestantes com sífilis, e } \\
\text { que é muito importante à abordagem dos parceiros nas consultas de pré-natal. }\end{array}$ & VI.D. \\
\hline 10 & $\begin{array}{l}\text { Rocha } \\
\text { AFB, et } \\
\text { al., } 2019 .\end{array}$ & $\begin{array}{l}\text { Management of sexual } \\
\text { partners of pregnant } \\
\text { women with syphilis in } \\
\text { northeastern Brazil - a } \\
\text { qualitive study. }\end{array}$ & $\begin{array}{l}\text { Estudo } \\
\text { Qualitativ } \\
\quad 0 .\end{array}$ & $\begin{array}{l}\text { O estudo consiste em relatos de profissionais de saúde de uma unidade básica do } \\
\text { Brasil, mostrando que os profissionais estão despreparados para fazer a abordagem do } \\
\text { parceiro da gestante com sífilis. Propõe a estratégia de incluir o parceiro no pré-natal, } \\
\text { não apenas no tratamento. }\end{array}$ & VI.D. \\
\hline 11 & $\begin{array}{l}\text { Cardoso } \\
\text { ARP, et } \\
\text { al., } 2018 .\end{array}$ & $\begin{array}{l}\text { Análise dos casos de } \\
\text { sífilis gestacional e } \\
\text { congênita nos anos de } \\
2008 \text { a } 2010 \text { em } \\
\text { Fortaleza, Ceará, Brasil. }\end{array}$ & $\begin{array}{l}\text { Estudo } \\
\text { Transvers } \\
\quad \text { al. }\end{array}$ & $\begin{array}{l}\text { Estudo com dados epidemiológicos que abrangem a gestante com sífilis. O estudo } \\
\text { mostra também que se a gestante com sífilis e o recém-nascido não receberem } \\
\text { assistência adequada, pode aumentar os casos de natimortos e abortos. }\end{array}$ & VI.D. \\
\hline 12 & $\begin{array}{l}\text { Magalhãe } \\
\text { s DMS, et } \\
\text { al., } 2013 .\end{array}$ & $\begin{array}{l}\text { Sífilis materna e } \\
\text { congênita: ainda um } \\
\text { desafio. }\end{array}$ & $\begin{array}{l}\text { Estudo } \\
\text { Descritivo } \\
\end{array}$ & $\begin{array}{l}\text { Estudo que mostra a relação do diagnóstico e tratamento com o histórico obstétricos } \\
\text { das gestantes. Além de mostrar que a sífilis congênita pode ser evitada se a gestante } \\
\text { com sífilis tiver realizado o pré-natal completo com as medidas de controle e prevenção } \\
\text { realizadas, e se o parceiro da gestante for incluído no pré-natal e no tratamento da sífilis. }\end{array}$ & VI.D. \\
\hline
\end{tabular}

REAS/EJCH | Vol.12(11) | e4741 | DOI: https://doi.org/10.25248/reas.e4741.2020 Página 6 de 12 


\begin{tabular}{|c|c|c|c|c|c|}
\hline № & $\begin{array}{l}\text { Autores/ } \\
\text { Ano de } \\
\text { publicaç } \\
\text { ão }\end{array}$ & Título da publicação & Método & Principais resultados/ Conclusões & $\begin{array}{c}\text { Nível de } \\
\text { evidência e grau } \\
\text { de } \\
\text { recomendação* }\end{array}$ \\
\hline 13 & $\begin{array}{l}\text { Campos } \\
\text { ALA, et } \\
\text { al., } 2012 .\end{array}$ & $\begin{array}{l}\text { Sífilis em parturientes: } \\
\text { aspectos relacionados ao } \\
\text { parceiro sexual. }\end{array}$ & $\begin{array}{l}\text { Estudo } \\
\text { Quantitati } \\
\text { vo. }\end{array}$ & $\begin{array}{l}\text { O estudo fez uma pesquisa sociodemográfica acerca dos parceiros das gestantes, que } \\
\text { mostra baixa renda, baixa escolaridade e muitos possuíam relações extraconjugais (o } \\
\text { que era sabido pela maioria das gestantes). Estudo mostra a importância de incluir o } \\
\text { parceiro no pré-natal e no tratamento da sífilis junto com a gestante, pois a maior } \\
\text { dificuldade da gestante em receber o tratamento adequado é o não tratamento do } \\
\text { parceiro. }\end{array}$ & VI.D. \\
\hline 14 & $\begin{array}{l}\text { Campos } \\
\text { ALA, et } \\
\text { al., } 2010 .\end{array}$ & $\begin{array}{l}\text { Epidemiologia da sífilis } \\
\text { gestacional em Fortaleza, } \\
\text { Ceará, Brasil: um agravo } \\
\text { sem controle. }\end{array}$ & $\begin{array}{c}\text { Estudo } \\
\text { Transvers } \\
\text { al e } \\
\text { descritivo. }\end{array}$ & $\begin{array}{l}\text { O estudo indica que a sífilis congênita só terá uma diminuição significativa quando as } \\
\text { medidas de prevenção e controle forem aplicadas de forma correta, já que apenas as } \\
\text { consultas de pré-natal não são suficientes para a prevenção. }\end{array}$ & VI.D. \\
\hline 15 & $\begin{array}{l}\text { Arnesen } \\
\text { L, et al., } \\
2015 .\end{array}$ & $\begin{array}{l}\text { Gestational syphilis and } \\
\text { stillbirth in the Americas: a } \\
\text { systematic review and } \\
\text { meta-analysis. }\end{array}$ & $\begin{array}{l}\text { Revisão } \\
\text { sistemátic } \\
\text { a e } \\
\text { metanális } \\
\quad \text { e. }\end{array}$ & $\begin{array}{l}\text { O estudo compara diversas pesquisas, e nele, conclui-se que o tratamento adequado é } \\
\text { muito eficaz para prevenção da sífilis congênita e suas consequências, já que a sífilis } \\
\text { não tratada aumenta a possibilidade perda fetal e natimorto. }\end{array}$ & I.A. \\
\hline 16 & $\begin{array}{l}\text { Ham DC, } \\
\text { et al., } \\
2015 .\end{array}$ & $\begin{array}{l}\text { Improving global } \\
\text { estimates of syphilis in } \\
\text { pregnancy by diagnostic } \\
\text { test type: A systematic } \\
\text { review and meta-analysis. }\end{array}$ & $\begin{array}{l}\text { Revisão } \\
\text { sistemátic } \\
\text { a e } \\
\text { metanális } \\
\quad \text { e. }\end{array}$ & $\begin{array}{l}\text { Realizado um estudo com dados mundiais, utilizando como base os exames de } \\
\text { treponema pallidum e VDRL. O estudo relata que para haver transmissão vertical da } \\
\text { sífilis, a mãe deve ter a infecção ativa, o que depende do estágio da doença, histórico } \\
\text { prévio, tratamento e exames laboratoriais (treponêmico e não treponêmico) }\end{array}$ & I.A. \\
\hline 17 & $\begin{array}{l}\text { Domingue } \\
\text { s RMSM, } \\
\text { et } \\
\text { al.,2014. } \\
\end{array}$ & $\begin{array}{l}\text { Strategies of testing for } \\
\text { syphilis during pregnancy. }\end{array}$ & $\begin{array}{l}\text { Estudo de } \\
\text { coorte. }\end{array}$ & $\begin{array}{l}\text { Estudo que avaliou a efetividade da triagem da sífilis no pré-natal. Abrange os riscos de } \\
\text { eventos adversos a gestação que podem ocorrer com início do pré-natal tardio. Fala do } \\
\text { teste rápido de sífilis, que deve ser realizado pela gestante e pelo parceiro, de } \\
\text { preferência, na primeira consulta de pré-natal. }\end{array}$ & IV.C. \\
\hline 18 & $\begin{array}{l}\text { Qin JB, et } \\
\text { al., } 2013 .\end{array}$ & $\begin{array}{l}\text { Risk factors for congenital } \\
\text { syphilis and adverse } \\
\text { pregnancy outcomes in } \\
\text { offspring of women with } \\
\text { syphilis in Shenzhen, } \\
\text { China: a prospective } \\
\text { nested case-control study. }\end{array}$ & $\begin{array}{l}\text { Estudo } \\
\text { caso } \\
\text { controle. }\end{array}$ & $\begin{array}{l}\text { O estudo abrange a importância de iniciar o pré-natal no início da gestação, e a } \\
\text { realização da triagem da sífilis nas primeiras consultas do pré-natal. }\end{array}$ & V.C. \\
\hline
\end{tabular}

REAS/EJCH | Vol.12(11) | e4741 | DOI: https://doi.org/10.25248/reas.e4741.2020 Página 7 de 12 


\section{Revista Eletrônica Acervo Saúde / Electronic Journal Collection Health | ISSN 2178-2091}

\begin{tabular}{|c|c|c|c|c|c|}
\hline № & $\begin{array}{l}\text { Autores/ } \\
\text { Ano de } \\
\text { publicaç } \\
\text { ão }\end{array}$ & Título da publicação & Método & Principais resultados/ Conclusões & $\begin{array}{l}\text { Nível de } \\
\text { evidência e grau } \\
\text { de } \\
\text { recomendação* }\end{array}$ \\
\hline 19 & $\begin{array}{l}\text { Hawkes } \\
\text { SJ,et al., } \\
2013 .\end{array}$ & $\begin{array}{l}\text { Early antenatal care: does } \\
\text { it make a difference to } \\
\text { outcomes of pregnancy } \\
\text { associated with syphilis? } \\
\text { A systematic review and } \\
\text { meta-analysis. }\end{array}$ & $\begin{array}{l}\text { Revisão } \\
\text { sistemátic } \\
\text { a e } \\
\text { metanális } \\
\text { e. }\end{array}$ & $\begin{array}{l}\text { O estudo mostra que o tratamento da sífilis é mais eficaz no primeiro trimestre de } \\
\text { gestação, já no segundo e terceiro trimestre aumentam os riscos para que ocorra a } \\
\text { transmissão vertical da sífilis e para eventos adversos, como morte fetal. }\end{array}$ & I.A. \\
\hline 20 & $\begin{array}{l}\text { Gomez } \\
\text { GB, et al., } \\
2013 .\end{array}$ & $\begin{array}{l}\text { Untreated maternal } \\
\text { syphilis and adverse } \\
\text { outcomes of pregnancy: a } \\
\text { systematic review and } \\
\text { meta-analysis. }\end{array}$ & $\begin{array}{l}\text { Revisão } \\
\text { sistemátic } \\
\text { a e } \\
\text { metanális } \\
\text { e. }\end{array}$ & $\begin{array}{l}\text { O estudo mostra que a sífilis gestacional aumenta os riscos de eventos adversos na } \\
\text { gravidez. A gestante com sífilis tem maiores chances de ocorrer aborto e natimorto do } \\
\text { que em gestantes sem sífilis. }\end{array}$ & I.A. \\
\hline 21 & $\begin{array}{l}\text { Galvão } \\
\text { TF, et al., } \\
2013 .\end{array}$ & $\begin{array}{l}\text { Safety of benzathine } \\
\text { penicillin for preventing } \\
\text { congenital syphilis: a } \\
\text { systematic review. }\end{array}$ & $\begin{array}{l}\text { Revisão } \\
\text { sistemátic } \\
\text { a de } \\
\text { ensaios } \\
\text { clínicos } \\
\text { randomiz } \\
\text { ados. }\end{array}$ & $\begin{array}{l}\text { Estudo mostra que as reações adversas do tratamento da sífilis com penicilina } \\
\text { benzatina em mulheres grávidas são muitos baixos, e que, não há justificativa para } \\
\text { utilizar outro tratamento em gestantes devido às preocupações de reações adversas. }\end{array}$ & III.C. \\
\hline 22 & $\begin{array}{l}\text { Hawkes } \\
\text { SJ, et al., } \\
2011 .\end{array}$ & $\begin{array}{l}\text { Effectiveness of } \\
\text { interventions to improve } \\
\text { screening for syphilis in } \\
\text { pregnancy: a systematic } \\
\text { review and meta-analysis. }\end{array}$ & $\begin{array}{l}\text { Revisão } \\
\text { sistemátic } \\
\text { a e } \\
\text { metanális } \\
\text { e. }\end{array}$ & $\begin{array}{l}\text { O estudo mostra que o início do pré-natal no primeiro trimestre de gestação pode ter } \\
\text { menores chances de eventos adversos da sífilis, pois se pode iniciar o quanto antes a } \\
\text { triagem da sífilis, já o pré-natal com início tardio (segundo ou terceiro trimestre) } \\
\text { aumentam as chances de eventos adversos. }\end{array}$ & I.A. \\
\hline
\end{tabular}

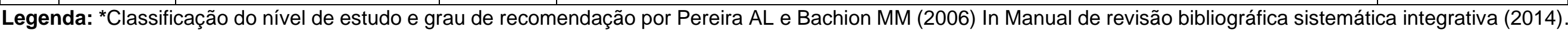
Fonte: Silveira CR, et al., 2020. 


\section{DISCUSSÃO}

A maioria dos estudos utilizados para a pesquisa associaram a sífilis gestacional com baixa escolaridade, cor de pele parda ou negra, religião católica, idade de 18 a 25 anos e início tardio do pré-natal $(3,4,11,16$ 20). Além disso, alguns estudos associaram a sífilis gestacional ao uso de drogas dos parceiros, baixa adesão ao pré-natal por parte das gestantes, e a vulnerabilidade social (artigos $3,4,11,17,18,20$ ). Além de que, um estudo mostrou que as práticas sexuais inseguras é um fator de risco para a sífilis presente na maioria das gestantes, como o não uso de preservativo e mais de um parceiro sexual (artigo 4).

A baixa escolaridade está associada ao aumento de infecções sexualmente transmissíveis devido à falta de informações, o que limita a gestante um baixo conhecimento dos cuidados em saúde, que diminui suas medidas de prevenção de doenças (FONTES MB, et al., 2017).

Um dos estudos, realizado no Brasil por Macedo VC, et al. (2017), mostrou que quanto mais precoce for o início da vida sexual da mulher, maior será sua promiscuidade sexual, e que quanto maior for a escolaridade, menor será o número de parceiros sexuais da mesma, diminuindo as chances de adquirir doenças sexualmente transmissíveis como a sífilis. Devido a isso, podemos associar à ocorrência da sífilis mais prevalente em mulheres com baixa escolaridade, condições de vida desfavoráveis e idade materna menor de 20 anos (artigo 4).

O pré-natal deve ser iniciado, de preferência, no primeiro trimestre da gestação com teste rápido na primeira consulta, pois se reagente, deve-se iniciar o tratamento o quanto antes, o que diminui os riscos de eventos adversos para a gestação (artigos 1,7,15,18,19,22). O tratamento mais indicado é 7.200.000Ul de penicilina benzatina, em 3 doses, sendo uma por semana, para a gestante e o parceiro (artigo 16). Não há estudos que comprovem que esse tratamento oferece riscos a gestantes, apenas em caso de alergia a penicilina (artigo 29).

Um dos estudos, realizado em Ghana por Dassah ET, et al. (2015), mostrou que o pré-natal no serviço particular pode ser um fator de risco para a sífilis gestacional, por ter chances de não ser realizada a triagem para sífilis.Já um dos estudos, realizado no Brasil por Domingues RMSM, et al.

(2014) entre 2011 e 2012, apontam que o pré-natal no serviço privado possui uma cobertura de testagem da sífilis elevada em relação ao pré-natal do serviço público, este estudo ainda mostra que no serviço privado a carteira de gestante nem sempre é disponibilizada, já que o pré-natal e a assistência ao parto são realizados pelo mesmo profissional. Mas, a gestante não possuir a carteirinha de pré-natal pode dificultar a continuidade do cuidado caso se tenha a procura em outro serviço (BRASIL, 2005).

Os estudos que focaram no tratamento da sífilis gestacional mostraram que o tratamento é mais eficaz na sífilis primária e secundária, por terem um declínio de titulação maior do que na sífilis terciária e latente (artigos $11,15,16)$.

A sífilis primária e secundária é caracterizada por sinais e sintomas presentes (como feridas, manchas, febre, etc.) sendo considerada uma infecção ativa, recente e de maior titulação. A sífilis terciária pode surgir até 40 anos após a infecção, e pode ter sintomas cardiovasculares e neurológicos, já a sífilis latente é considerada uma infecção assintomática, e de baixa titulação (BRASIL, 2010)

A titulação da sífilis é feita a partir do exame de VDRL (Venereal Diseases Research Laboratory) que é considerado o exame de rastreio mais importante do primeiro trimestre de gestação, identificando se a doença é ativa ou não. Quanto ao teste rápido e o exame treponemo, do treponema pallidum, indicam se a pessoa já teve contato com sífilis, pelos anticorpos da bactéria presentes, mas não é um indicativo de doença ativa, já que é sempre reagente, mesmo após o tratamento (BRASIL, 2016).

Existem casos em que a gestante pode apresentar o exame de VDRL reagente, com uma titulação baixa ou alta, e o teste de anticorpos e/ou teste rápido não reagente, que se caracteriza como sífilis falso positivo, o fenômeno prozona. Esse fenômeno pode ocorrer, entre outros fatores, pelo aumento dos anticorpos da pessoa, em que ela apresenta uma titulação no exame do VDRL, mas ela não tem a doença por não ter anticorpos da bactéria treponema pallidum presente. 
A sífilis falsa positiva, caso supracitado, a literatura aponta que não há necessidade de tratamento por oferecer resultados enganosos, o que pode dificultar no controle e rastreamento da sífilis (artigo 8). Segundo o MS, a assistência ao pré-natal adequada a gestante consiste em: realização de no mínimo seis consultas de pré-natal, realização do teste rápido e exames específicos como o VDRL na primeira consulta e um segundo teste a partir da $28^{\circ}$ semana; e se positivo, realizar a abordagem e o tratamento do parceiro (BRASIL, 2016).

De acordo com as Diretrizes para o controle da Sífilis Congênita do MS (2006), o tratamento da sífilis materna é considerado inadequado quando o parceiro não faz o tratamento ou o faz de forma incompleta. 0 que evidencia a importância da participação do parceiro nas consultas.

Em relação ao tratamento da gestante e seu parceiro sexual, os estudos mostraram que na maioria dos casos de sífilis gestacional, o tratamento é considerado inadequado pelo não tratamento do parceiro sexual mesmo que a gestante tenha realizado o esquema de tratamento completo (artigos 13 e 14), já que o tratamento do parceiro é determinante para a cura da gestante (artigo 1)

Em relação ao papel do enfermeiro na abordagem do parceiro sexual da gestante com sífilis, muitos dos estudos mostraram que os profissionais encontram muita dificuldade na abordagem (artigos 10,18,19) principalmente porque a gestante pode ter vários parceiros sexuais e porque o homem relaciona os cuidados da gestação apenas à mulher, não entendendo os riscos do não tratamento da parte dele.

Em um dos estudos realizado no Brasil, mostrou que a recusa do tratamento por parte parceiro está relacionada ao medo de injeções e por não acreditarem que estão doentes e também evidenciou relatos dos profissionais de saúde, que informaram desconhecer o protocolo e diretrizes do MS, e que a abordagem do parceiro não é realizada, apenas prescrevem o tratamento e pedem a gestante entregar ao seu parceiro (artigos 10 e 13).

De acordo com uma das pesquisas realizadas no Brasil, na maioria dos casos de sífilis gestacional, a própria gestante comunica o parceiro do diagnóstico de sífilis, e entre as que não revelaram o diagnóstico ao parceiro, a maioria é resultado do desconhecimento da importância do tratamento do mesmo por parte da gestante ou por não estar mais com o mesmo parceiro. Além disso, o estudo aborda que o diagnóstico de doenças sexualmente transmissíveis pode ser relacionado a questões de infidelidade, o que dificulta a abordagem e o tratamento do parceiro sexual (artigo 10).

Os resultados sugerem que os profissionais de saúde devem ser capacitados em relação ao atendimento nos casos suspeitos e positivos quanto à sífilis gestacional, para que os mesmos estejam preparados para lidar com os casos da forma recomendada pelo MS, e principalmente, para que saibam abordar os parceiros das gestantes para que eles compreendam a importância de eles participarem do pré-natal e de fazerem o tratamento.

Os estudos mostraram que os profissionais precisam promover ações que auxiliem no diagnóstico e no controle da doença, como executar busca ativa efetiva dos pacientes com diagnóstico de sífilis, fazer acompanhamento dos casos em tratamento e realizar a notificação dos casos conforme Protocolo Clínico e Diretrizes Terapêuticas para atenção integral às pessoas com Infecções Sexualmente Transmissíveis (IST) do MS (BRASIL, 2019; OLIVEIRA DR e FIGUEIREDO MSN, 2011).

De acordo com a Nota Informativa no 02-SEI/2017 do MS (2017), todas as mulheres sintomáticas ou assintomáticas, com resultado reagente no teste treponêmico ou não treponêmico de qualquer titulação durante o pré-natal, parto ou puerpério devem ser notificadas como sífilis gestante.

O papel do enfermeiro nesses casos é essencial, pois ele é um dos profissionais de saúde responsável por fazer o acompanhamento de pré-natal, assim, identificando os casos de sífilis, e realizando a orientação a gestante e o parceiro sobre o tratamento adequado, modo de transmissão e consequências para a gestação e para o recém-nascido (artigo 1). 


\section{CONSIDERAÇÕES FINAIS}

Pode-se concluir que exames de triagem da sífilis no pré-natal devem se iniciar no primeiro trimestre, com o teste rápido na primeira consulta, pois quanto antes a gestante e o parceiro tratarem, menores serão os riscos de eventos adversos na gestação. Evidenciou-se que o pré-natal das gestantes com exames de sífilis positivos é mais efetivo se houver a inclusão do parceiro. O enfermeiro tem um papel essencial no acompanhamento da gestante com sífilis, é o profissional que tem capacitação para iniciar a triagem de sífilis no pré-natal, incluindo o teste rápido na primeira consulta e fazer as orientações de educação em saúde. Devem ser realizados mais estudos e pesquisas sobre o papel do enfermeiro na abordagem dos parceiros sexuais no pré-natal e tratamento das gestantes com sífilis, pois é um assunto com pouca abordagem na literatura.

\section{REFERÊNCIAS}

1. ARNESEN L, et al. Gestational syphilis and stillbirth in the Americas: a systematic review and meta-analysis. 2015. Rev PanamSalud Publica. 2015;37(6):422.

2. AVELLEIRA JCR. BOTTINO G. Sífilis: diagnóstico, tratamento e controle. AnBrasDermatol. 2006;81(2):111-126.

3. BRASIL. Ministério da Saúde, Secretaria de Atenção à Saúde, departamento de ações programáticas estratégicas. pré-natal e puerpério atenção qualificada e humanizada manual técnico. Brasília, 2005.

4. BRASIL. Ministério da Saúde, Secretaria de Vigilância em Saúde, Programa Nacional de DST e Aids. Diretrizes para o Controle da Sífilis Congênita. Brasília, 2006.

5. BRASIL. Ministério da Saúde. Protocolos da Atenção Básica: Saúde das Mulheres / Ministério da Saúde, Instituto Sírio-Libanês de Ensino e Pesquisa - Brasília: Ministério da Saúde, 2016.

6. BRASIL. Ministério da Saúde. Secretaria de Vigilância Em Saúde Departamento de Dst, Aids e Hepatites Virais. SíFILIS Estratégias para Diagnóstico no Brasil. [internet]. 2010. [citado 2019 mai 9]. Disponível em: <http://bvsms.saude.gov.br/bvs/publicacoes/sifilis_estrategia_diagnostico_brasil.pdf>.

7. BRASIL. Ministério da Saúde. Secretaria de Vigilância em Saúde. Departamento de Vigilância, Prevenção e Controle das Doenças Sexualmente Transmissíveis, Aids e Hepatites Virais. Manual Técnico para Diagnóstico da Sífilis. Brasília: Ministério da Saúde, 2016.

8. BRASIL. Nota Informativa no 02-SEI/2017 - DIAHV/SVS/MS. Altera os critérios de definição de casos para notificação de Sífilis Adquirida, Sífilis em Gestantes e Sífilis Congênita. Brasília, 13 de outubro de 2017.

9. BRASIL. Secretaria de Vigilância Em Saúde, Ministério da Saúde. Boletim Epidemiológico de Sífilis - 2018. Brasília, 2018.

10. Brasil. Ministério da Saúde. Secretaria de Vigilância em Saúde. Departamento de Doenças de Condições Crônicas e Infecções Sexualmente Transmissíveis. Protocolo Clínico e Diretrizes Terapêuticas para Atenção Integral às Pessoas com Infecções Sexualmente Transmissíveis (IST)/Ministério da Saúde, Secretaria de Vigilância em Saúde, Departamento de Doenças de Condições Crônicas e Infecções Sexualmente Transmissíveis. - Brasília: Ministério da Saúde, 2019. 248 p.

11. CAMPOS ALA, et al. Epidemiologia da sífilis gestacional em Fortaleza, Ceará, Brasil: um agravo sem controle. Cad. Saúde Pública. 2010;26(9):1747-1755.

12. CAMPOS ALA, et al. Sífilis em parturientes: aspectos relacionados ao parceiro sexual. RevBrasGinecol Obstet. 2012;34(9):397-402.

13. CARDOSO ARP, et al. Análise dos casos de sífilis gestacional e congênita nos anos de 2008 a 2010 em Fortaleza, Ceará, Brasil.2015. Ciênc saúde coletiva. 2018;23(2):563-574.

14. CUNHA AE, et al. Importância do pré-natal na prevenção da Sífilis Congênita. Rev Para Med. 2006;20(1):47-51.

15. DASSAH ET, et al. Factors associated with failure to screen for syphilis during antenatal care in Ghana: a case control study. I BMC Infect Dis. 2015;(13)15:125.

16. DOMINGUES RMSM, et al. Prevalência de sífilis na gestação e testagem pré-natal: Estudo Nascer no Brasil. Rev Saúde Pública. 2014;48(5):766-774.

17. FIGUEIREDO MSNG, et al. Percepção de enfermeiros sobre a adesão ao tratamento dos parceiros de gestantes com sífilis. Rev Rene. 2015;16(3):345-54.

18. FONTES MB, et al. Fatores determinantes de conhecimentos, atitudes e práticas em DST/Aids e hepatitesvirais, entre jovens de 18 a 29 anos, no Brasil. Ciênc. saúdecoletiva. 2017;22(4):1343-1352.

19. GALVÃO TF, et al. Safety of Benzathine Penicillin for Preventing Congenital Syphilis: A Systematic Review. PLoS One. 2013;8(2):e56463.

20. GOMEZ GB, et al. Untreated maternal syphilis and adverse outcomes of pregnancy: a systematic review and metaanalysis. Bull World Health Organ. 2013;1;91(3):217-226.

21. HAM DC, et al. Improving global estimates of syphilis in pregnancy by diagnostic test type: A systematic review and meta-analysis. Int J Gynaecol Obstet. 2015;1:S10-4.

22. HAWKES SJ, et al. Early Antenatal Care: Does It Make a Difference to Outcomes of Pregnancy Associated with Syphilis? A Systematic Review and Meta-Analysis. PLoS One. 2013;8(2):e56713. 
23. HAWKES SJ, et al. Effectiveness of interventions to improve screening for syphilis in pregnancy: a systematic review and meta-analysis. Lancet Infect Dis. 2011;(9):684-91.

24. LIU LL, et al. Incidence and risk factors for the prozone phenomenon in serologic testing for syphilis in a large control. ClinInfectDis. 2014 Aug 1;59(3):384-9.

25. MACEDO VC, et al. Fatores de risco para sífilis em mulheres: estudo caso-controle. Rev Saúde Publica. $2017 ; 51: 78$.

26. MAGALHÃES DMS, et al. Sífilis materna e congênita: ainda um desafio. Cad Saúde Pública. 2013;29(6):1109-1120.

27. MARTHA WFR, et al. Maternal Titers After Adequate Syphilotherapy During Pregnancy. Clin Infect Dis. 2015;(60):686-690.

28. MENDES KDS, et al. Revisão integrativa: método de pesquisa para a incorporação de evidências na saúde e na enfermagem. Texto \& contexto - enferm. 2008;17(4):758-764.

29. NASCIMENTO MI, et al. Gestações complicadas por sífilis materna e óbito fetal. 2011.. RevBrasGinecol Obstet. 2012;34(2):56-62.

30. NONATO SM, et al. Sífilis na gestação e fatores associados à sífilis congênita em Belo Horizonte-MG, $2010-2013$. EpidemiolServ Saúde. 2015; 24(4):681-694.

31. OLIVEIRA DR, FIGUEIREDO MSN. Abordagem conceitual sobre a sífilis na gestação e o tratamento de parceiros sexuais. RevCofen. 2011;2(2):108-111.

32. PEREIRA AL, BACHION MM. Atualidades em revisão sistemática de literatura, critérios de força e grau de recomendação de evidência. Rev Gaúcha Enferm. 2006;27(4):491, 2006. In: Manual de revisão bibliográfica sistemática integrativa: a pesquisa baseada em evidencias. Grupo Ănima Educação. 2014.

33. QIN JB, et al. Risk Factors for Congenital Syphilis and Adverse Pregnancy Outcomes in Offspring of Women With Syphilis in Shenzhen, China: A Prospective Nested Case-Control Study. Sex Transm Infect. 2013;41(1):13-23.

34. ROCHA AFB, et al. Management of sexual partners of pregnant women with syphilis in northeastern Brazil - a qualitative study. BMC Health Services Research. 2019;19:65.

35. SECRETARIA DE ESTADO DA SAUDE SES-SP, Serviço de Vigilância Epidemiológica Coordenação do Programa Estadual DST/Aids-SP, Coordenadoria de Controle de Doenças - CCD. Sífilis congênita e sífilis na gestação. Rev Saúde Pública. 2008;42(4):768-72.

36. SILVA AM, et al. Factores Asociados Con Falla en el Diagnostico y Tratamiento de Sifilis Materna. Medlnfant. 2016;23(4):293-298, diciembre 2016.

37. VASCONCELOS MIO, et al. Estratégias e Desafios dos Enfermeiros da Atenção Básica para o Tratamento Simultâneo da Sífilis. RevBraspromoç saúde. 2016;2:1584-1591. 